\title{
Exploring the perceptual spaces of faces, cars and birds in children and adults
}

\author{
James W. Tanaka, Tamara L. Meixner, and Justin Kantner \\ Cognition and Brain Sciences Program, Department of Psychology, University of Victoria, \\ Canada
}

\begin{abstract}
While much developmental research has focused on the strategies that children employ to recognize faces, less is known about the principles governing the organization of face exemplars in perceptual memory. In this study, we tested a novel, child-friendly paradigm for investigating the organization of face, bird and car exemplars. Children ages 3-4, 5-6, 7-8, 9-10, 11-12 and adults were presented with 50/50 morphs of typical and atypical face, bird and car parent images. Participants were asked to judge whether the 50/50 morph more strongly resembled the typical or the atypical parent image. Young and older children and adults showed a systematic bias to the atypical faces and birds, but no bias toward the atypical cars. Collectively, these findings argue that by the age of 3 , children encode and organize faces, birds and cars in a perceptual space that is strikingly similar to that of adults. Category organization for both children and adults follows Krumhansl's (1978) distance-density principle in which the similarity between two exemplars is jointly determined by their physical appearance and the density of neighboring exemplars in the perceptual space.
\end{abstract}

\section{Introduction}

Much of the research examining age-related changes in face processing has focused on the kind of information and strategies that children employ to recognize faces (Carey \& Diamond, 1994; Mondloch, Le Grand \& Maurer, 2002; Pellicano \& Rhodes, 2003; Sangrigoli \& de Schonen, 2004; Tanaka, Kay, Grinnell, Stansfield \& Szechter, 1998a). This line of investigation has contributed important findings toward our understanding of the developmental changes governing the recognition of individual faces. However, less is known about the way that children organize individual faces in perceptual memory. To address this question, we compared the perceptual structure of children and adults for three types of object categories - faces, birds and cars. We employed a child-friendly similarity task in which 50/50 morphs created from typical and atypical parent images of faces, birds and cars were presented to participants. To probe the category structure for these objects, child and adult participants were asked to judge whether the 50/50 morph more strongly resembled the typical or the atypical parent image. We found that for birds and faces, young children and adults judged the 50/50 morph as more similar to the atypical than the typical parent image. This atypicality bias suggests that the similarity judgments of very young children, like adults, are influenced by the structure, location and distribution of category exemplars in the perceptual space. 
It has been hypothesized that faces are organized in a multi-dimensional, psychological 'face space' that is defined by the perceptual dimensions of the face stimulus (e.g. face width, chin shape, nose length) (Valentine, 1991; for an updated version see Lewis, 2004). In this model, face typicality varies as a function of distance from the origin of the space. Typical or average looking faces are located near the center and less typical or more unusual looking faces are located in more peripheral regions. A critical assumption of the face space model asserts that faces are normally distributed such that there is a high density of typical faces at the origin and a decreasing proportion of atypical exemplars as distance from the origin increases. Consequently, typical faces are subject to heightened competition during recognition from neighboring typical face representations and are therefore assumed to be less distinctive in memory. In contrast, atypical faces have fewer neighbors, are exposed to less competition, and are therefore more distinctive and more memorable.

The empirical results are consistent with the distinctiveness claim. Bartlett, Hurry and Thorley (1984) and Going and Read (1974) demonstrated that photographs of highly unique faces were correctly recognized more often than faces of low uniqueness. Johnston and Ellis (1995) found that adults were faster and more accurate at recognizing atypical faces than typical faces, but were faster to categorize typical faces as a face than atypical ones. The atypicality face advantage has been demonstrated for the recognition of newly familiarized faces (Bartlett et al., 1984; Light, Kayra-Stuart \& Hollander, 1979; Vokey \& Read, 1992) and famous faces (Valentine \& Bruce, 1986). Rhodes, Byatt, Tremewan and Kennedy (1997) showed that face caricatures, in which the features of a face are exaggerated relative to the average face prototype, are recognized more readily than anti-caricatures, in which distinctive features of a face are diminished relative to the prototype. 1 In summary, the collective evidence supports the predictions of the face space model where the clustering of representations in face spaces renders typical faces less memorable and atypical faces more memorable in recognition.

An important question is the extent to which the structure of a child's face space changes over the course of development. Using multi-dimensional scaling (MDS) techniques and a novel odd-man-out paradigm, Nishimura, Maurer and Gao (2009) probed the face space organization of 8-year-old children and adults. A key finding was that the faces that appeared most similar to the adults were also judged as most similar by the 8-year-olds; however, 8-year-olds showed more variability in their judgments, indicating that their diagnostic criteria were less stable. Adults incorporated multiple face dimensions (e.g. eyes, face shape, expression, eyebrows) in their judgments, whereas young children relied more on a single dimension: the eyes. The authors concluded that despite relying on fewer dimensions, 8-year-olds nevertheless exhibit a face space structure that is remarkably similar to that of adults.

Other evidence indicates that at a relatively early age, children have begun to organize faces into an adult-like face space structure with regard to typicality. Chang, Levine and Benson (2002) found that 6-year-olds, like older children (8- and 10-year-olds) and adults, choose face caricatures as being more distinctive than the original face and their corresponding anticaricatures. The magnitude of the caricature effect exhibited by 6 -year-olds, however, was less than the level shown by older children and adults. These findings suggest that by 6 years of age, children have received sufficient exposure to faces to determine the averageness or unusualness of a face, but perhaps not enough experience to construct a face space that is as differentiated as the adults'.

\footnotetext{
${ }^{1}$ Prototype-based or norm-based models posit that the center of face space contains the average face of the population whereas exemplar-based models make no special claim about an average representation. While we are neutral on this issue, we adhere to the common assumption of both models that faces are normally distributed in the space according to their structural typicality.
} 
While it is clear that typicality relations are preserved by children, it is less certain whether the distribution (i.e. density) of exemplars in the face space is the same for children as it is for adults. Johnston and Ellis (1995) found that 5- to 7-year-olds were faster to classify a typical face as a face than an atypical face, but recognized typical faces as accurately as atypical faces. This latter finding is at odds with the density prediction that typical faces will be more difficult to identify than atypical faces due to the increased competition from nearby face representations. To account for their findings, Johnston and Ellis (1995) offered two possible accounts of face space: either the representations in a child's face space are uniformly distributed such that the distance to the nearest neighbor was the same for typical and atypical faces or, conversely, the face space is compressed to the point where the nearest neighbor effect between typical and atypical faces was eliminated. In both cases, it was speculated that the clustering of representations in a child's face space was distorted relative to the normal distribution of faces in the adult face space.

According to Krumhansl's (1978) distance-density hypothesis, the impact of exemplar density on perceived similarity can be expressed in the formula stated below:

$$
\text { Similarity }\left(\text { face }_{1}, \text { face }_{2}\right)=d\left(\text { face }_{1}, \text { face }_{2}\right)+\alpha \delta\left(\text { face }_{1}\right)+\beta \delta\left(\text { face }_{2}\right)
$$

where the psychological similarity is determined by the physical correspondence between face $_{1}$ and face $_{2}, d\left(\right.$ face $_{1}$, face $\left._{2}\right)$, the spatial density of exemplars surrounding face $1, \delta\left(\right.$ face $\left._{1}\right)$ and face $_{2}, \delta\left(\right.$ face $\left._{2}\right)$, and the relative weights, $\alpha$ and $\beta$, assigned to those densities. Density $(\delta)$ is calculated as the summed difference between the target exemplar and all exemplars in the stimulus domain such that near neighbors will have a larger impact on density than far neighbors. Critically, the distance-density hypothesis predicts that two faces that are equivalent to one another in their physical appearance will be perceptually more similar to each other if they are located in a sparse region of the face space than if they are situated in a denser region. This concept is instantiated in Lewis' (2004) Face-Space-R model where the recognition of a specific face is determined by the number and proximity of neighboring face exemplars in the space.

The influence of exemplar density on the perceived similarity of two faces can be represented computationally in an attractor field model. According to this approach, face exemplars attract perceptual inputs by carving out valleys or basins of activations in the similarity space (Tank \& Hopfield, 1987). In recognition, inputs decrease their computational energy by following the path that leads to the attractor basin of the nearest representation. The stimulus need not be a perfect fit with its underlying representation, only a close enough approximation to fall within the boundaries of the representation's attractor field. Thus, the potential activation of any given representation is proportional to the size or span of its attractor field, such that representations with large attractor fields will capture a broader range of stimulus inputs than representations with smaller attractor fields. Over the course of development and with perceptual experience, the size of the attractor fields can shrink in size as the face recognition system becomes more finely tuned to the specific features that distinguish individual faces. For example, Humphreys and Johnson (2007) found that 7-month-old infants can detect finer distinctions in a morph gradient than their 4month-old counterparts.

Given that typical faces cluster closer together in face space, it is assumed that their attractor fields will be more constricted than the attractor fields of atypical faces, which are less constrained by neighboring representations (see Figure 1). To test the prediction of the attractor field model, Tanaka and colleagues presented participants with faces that were 50/50 morphs between an atypical parent face and a typical parent face. Importantly, each 
50/50 face morph was an equal distance from its typical and atypical parent faces in geometric space (i.e. the $d$ parameter in the Krumhansl equation). As a result of being located in a sparser region of face space, the atypical face is predicted to have a larger attractor field than the typical face, and in turn capture a broader set of face inputs than the smaller attractor field of the typical face. Consistent with the attractor field account, participants judged the 50/50 morph as bearing a stronger resemblance to the atypical parent face than the typical parent face (Tanaka, Giles, Kremen \& Simon, 1998b), and were less able to discriminate subtle differences between morphs and atypical faces than morphs and typical faces (Tanaka \& Corneille, 2007). These predictions are compatible with computational models of face recognition demonstrating that typical and atypical faces differ in their relative discrimination and recognition (Lewis, 2004; Meytlis \& Sirovich, 2007; O'Toole, Deffenbacher, Valentin \& Abdi, 1994; Wilson, Loffler \& Wilkinson, 2002). Subsequent experiments also revealed an atypicality bias for bird and car objects, indicating that exemplars in these categories are normally distributed in psychological space in a manner that is similar to faces (Tanaka \& Corneille, 2007).

In the present study, we investigated the category density of typical and atypical faces, cars and birds. Following previous procedures (Tanaka et al., 1998; Tanaka \& Corneille, 2007), children (ages 3 through 12) and adults were asked to judge whether a 50/50 face, bird and car morph appeared more similar to its typical or atypical parent. An advantage of the similarity task is that it is simple enough for very young children to understand but still provides a sensitive measure of density relationships. To control for own-age effects in children (Anastasi \& Rhodes, 2005), we pilot tested photographs of children of 10 to 12 years and selected the faces that were rated highest and lowest in typicality.

As one of the contrast categories to faces, we selected birds, assuming that the child's experience would increase with age. For the other non-face category, we employed birds and cars from the 1990s that were tested in a previous experiment (Tanaka \& Corneille, 2007). Unlike birds, we expected category experience to cars of this vintage to show less change with age. We predicted that if children have not yet established density relations for cars, they should be equally likely to select the typical as the atypical parent. In contrast, if children are sensitive to the distribution of exemplars within the categories of faces, and, perhaps, birds, the 50/50 morph should be perceived as bearing a stronger likeness to the atypical than the typical parent.

\section{Method}

\section{Participants}

Six age groups were tested in this study. Twenty-two 3-4-year-olds ( 9 boys, 13 girls, mean age $=3.8$ ), 17 5-6-year-olds ( 5 boys, 12 girls, mean age $=5.6$ ), 25 7-8-year-olds ( 14 boys, 11 girls, mean age $=7.3$ ), $269-10$-year-olds ( 5 boys, 21 girls, mean age $=9.2$ ), 26 11-12year-olds ( 11 boys, 15 girls, mean age $=11.2$ ), and 33 undergraduates ( 26 females, 7 males, mean age $=23.1$ ) participated in the present experiment. Participants aged 3-4 attended a Montessori preschool located in Victoria, BC, participants aged 5-12 were tested during a free one-day camp held at the University of Victoria, and adult participants were students from the University of Victoria and completed the experiment for course credit.

\section{Materials}

Face stimuli were collected from the internet and varied in age between 10 and 12 years old. The faces were modified in Adobe Photoshop according to a standard procedure in which external features including hair, ears and neck were cropped with the selection tool set at a 'feather' parameter of 10 pixels. Faces had little or no hair covering their foreheads, no 
obvious facial blemishes, no glasses and depicted closed mouth, neutral expressions. All of the faces were displayed on a white background in a frontal or near-frontal viewing pose, and were $3.26^{\prime \prime} \times 4.3^{\prime \prime}$ at $72 \mathrm{dpi}(\mathrm{w} \times \mathrm{h})$ in size (see Figure 2$)$.

The atypical and typical faces were selected in the following manner. The 100 male child faces meeting the above criteria were presented in a slideshow for the duration of 5 seconds. Twelve participants were asked to rate the distinctiveness of each face on a scale of ' 1 ' to '7' with ' 1 ' representing very non-distinctive and ' 7 ' representing very distinctive. Participants were instructed to rate the faces according to how distinctive they appeared relative to the general population. From the original set of 100 faces, eight faces were selected on the basis of being rated as either very high or very low in typicality. The four chosen atypical faces had a mean distinctiveness rating of $5.85(S D=0.67)$ and the four typical faces had a mean distinctiveness rating of $2.14(S D=1.11)$. These eight faces were equated for luminance and contrast information with the SHINE (Spectrum, Histogram, and Intensity Normalization and Equalization) toolbox (Willenbockel, Sadr, Fiset, Horne, Gosselin \& Tanaka, in press). Using Abrosoft Fantamorph 4 a total of $1650 / 50$ face morphs were generated by averaging each atypical face with each typical face. Key points for facial features were kept relatively constant, with 5 points on the mouth, 4 points on each eye, 4 points on the nose, 3 points on each eyebrow, and 6 points for the outline of the face. Occasionally extra points were required to eliminate shadows.

Following a previous experiment by Tanaka and Corneille (2007), car stimuli were taken from the October 1994 issue of Road and Track magazine and were 3.47" $\times 2.08^{\prime \prime}$ at $72 \mathrm{dpi}$ $(\mathrm{w} \times \mathrm{h})$ and bird stimuli were taken from Peterson First Guides to Birds and were 2.53" $\times$ 2.53 " at 99 dpi. The four atypical birds (Brown-Headed Cowbird, Tricolored Heron, Great Black-backed Gull, Rubythroated Hummingbird) had a mean distinctiveness rating of 4.38 and the four typical birds (American Woodcock, Eastern Bluebird, Canada Warbler, Chipping Sparrow) had a mean distinctiveness of 1.78. The four atypical cars (1995 Mitsubishi 3000GT, 1995 Pontiac Firebird, 1995 Acura NSX, 1995 Mazda RX-7) had a mean distinctiveness rating of 4.22 and the four typical cars (1995 Buick Century, 1995 Chevrolet Caprice Classic, Impala SS, 1995 Subaru Impreza, 1995 Dodge Spirit) had a mean distinctiveness rating of 1.77. The atypical face, car and bird exemplars were morphed with the typical face, car and bird exemplars yielding a total of 16 face morphs, 16 car morphs and 16 bird morphs.

\section{Procedure}

Participants age 5 and older were tested on computers running the E-Prime software package. Each trial consisted of seeing a 1-second-long fixation cross followed by a typical and an atypical probe appearing simultaneously on the left and right sides of the screen. After a duration of 2 seconds, the morph of those two probes was added to the middle of the screen. The participant was then asked to decide whether he/she thought the probe image more strongly resembled the image on the left side of the screen or the right side of the screen. Child participants were run individually with the aid of a research assistant who was blind to the predictions of the study and to the typicality ratings of the stimuli. The child indicated his/her decision to their assistant either by pointing to the probe or by orally saying 'left' or 'right'. The assistant recorded decisions by pressing the appropriately labeled key. There was a 1-second intertrial interval. Undergraduate participants followed an identical procedure with the exception of having an assistant input their responses. For ease of testing, preschool age children were tested using booklets containing three images per page (the morph in the middle and the typical and atypical parents on the left and right side). The experimental trials were blocked according to category (faces, cars, birds) and the presentation order of the blocks was counterbalanced across participants. Each triplet (two parents and morph) was shown twice. There were 16 morphs, for a total of 32 experimental 
trials per category. The presentation order was random. The left and right positions of the typical and atypical probes were counterbalanced across trials. The child indicated his/her response by a pointing gesture. The 3-4- and 5-6-year-old children completed the bird and face tasks, but not the car task due to time constraints.

\section{Results}

The 50/50 morph was judged to be more similar to the atypical parent than to the typical parent for faces, birds and cars on $63 \%, 71 \%$ and $50 \%$ of the trials, respectively. The atypical face preference was reliably above the $50 \%$ unbiased level by participants $[t(148)=$ $13.89, p<.01]$ and by items $[t(15)=2.31, p<.05]$. The preference for the atypical bird was significantly above the $50 \%$ level by participants $[t(148)=18.27, p<.01]$ and by items $[t(15)=7.90, p<.01]$. The preference for the atypical car did not reliably differ from the $50 \%$ unbiased level by participants $[t(109)=-.356, p>.05]$ or by item. $[t(15)=.224, p>$. 05].

We examined the percentage of atypicality responses as a function of age and category for faces and birds, excluding the car category which was not tested with the 3-4- and 5-6-yearold children. As shown in Figure 3, each age group showed a reliable preference for the atypical face and atypical bird exemplar above the 50\% level, $p<.05$. An analysis of variance was performed with category (faces, birds) as the within-subjects factor and age $(3-4,5-6,7-8,9-10,11-12$, adults) as the between-groups factor in a mixed factorial design. The results revealed a main effect of category, $F(1,143)=41.512, p<.01, R^{2}=$. 225 , in which the atypicality bias to birds was greater than the atypicality bias to faces. Neither the main effect of age, $F(5,143)=1.667, p>.05, R^{2}=.055$, nor the interaction between category and age, $F(4,143)=1.089, p>.05, R^{2}=.037$, was significant. To explore possible age-related trends, correlational analyses were performed with age as a categorical variable $(0=3-4$ years, $1=5-6$ years, etc. $)$ and the atypicality bias for birds and faces as continuous variables. While the correlation between age and the atypicality bias for birds was not significant, $r=-.02, p=.79$, the correlation between age and the atypicality bias for faces reached a reliable level, $r=0.21, p=.01$.

In a second ANOVA, we examined all three categories (cars, faces, and birds) but excluded the 3-4- and 5-6-year-old age groups. This analysis showed a significant main effect of category $F(2,212)=95.082, p<.01, R^{2}=0.473$, and again, neither the main effect of age, $F(3,106)=1.322, p>.05, R^{2}=.036$, nor the age by category interaction, $F(6,212)=1.015$, $p>.05, R^{2}=.028$, was significant. Subsequent tests on the main effect of category revealed that the atypicality bias was greater to birds (73\%) than to faces (65\%) and cars $(50 \%)$ and was greater to faces than cars, $p<.05$.

\section{Discussion}

In this study, we employed a similarity task to investigate the perceptual space of faces, birds and cars in five groups of children with ages of 3-4, 5-6, 7-8, 9-10 and 11-12 years and a group of adult participants. Both the child and adult groups exhibited a reliable atypicality bias for faces, where the 50/50 face morph was judged as appearing more similar to the atypical parent face than the typical parent face. The magnitude of the atypicality bias remained robust across the age groups such that the preference for the atypical face was evident for 3-4-year-old children $(M=57 \%)$, and as strong for 5-6-year-old children $(M=$ $62 \%$ ) as it was for older children (7- and 8-year-olds: $M=64 \%$; 9 - and 10-year-olds: $M=$ $65 \%$; 11 - and 12-year-olds: $M=64 \%)$ and adults $(M=66 \%)$. This is compelling evidence that very young children organize typical and atypical faces in a perceptual space that is strikingly similar to adults. Although there was a small but reliable correlation between age 
and the atypicality bias for faces $(r=.21)$, our results suggest that the face space of very young children approximates the mature face space organization shown by adults (Crookes \& McKone, 2009).

The present atypicality results appear to be at odds with the previous findings where young children were no better at recognizing atypical faces than typical faces (Johnston \& Ellis, 1995). According to a face space model, atypical faces should show a retrieval advantage owing to their location in a sparser region of perceptual space where there is less competition for recognition. There are several reasons that might account for the apparent discrepancy between the current experiment and the Johnston and Ellis study. First, the faces tested in the Johnston and Ellis study were typical and atypical adult faces whereas the faces used in the present experiment were typical and atypical child faces. As supported by the own-age findings (Anastasi \& Rhodes, 2005), young children may have a differentiated perceptual space for child faces, but not for adult faces. Second, the Johnston and Ellis study used a short-term memory task with a fairly high memory load (i.e. simultaneous presentation of nine atypical and nine typical faces). The relatively poor memory performance of the 5-year-old group $\left(d^{\prime}=\sim .60\right)$ might have masked possible typicality differences. In contrast, the current paradigm incurred no memory load and was a test of direct perception. Finally, although the current results indicate clear influences of typicality on perception, it is possible that there is a developmental lag before these distinctions are realized as a recognition advantage for atypical faces.

It is worth noting that the atypicality bias was not restricted to the face category. When presented with 50/50 bird morphs, children and adult participants selected the atypical bird parent over the typical bird parent on $72 \%$ of the trials, indicating that they were sensitive to the structure and typicality of the birds. It is not surprising that young children are sensitive to structural variations in object categories given that previous studies have shown that infants form prototypes at an early age (Quinn, 1987, 2002; Strauss, 1979), look significantly longer at more typical items than less typical items (Southgate $\&$ Meints, 2000), and learn the names of typical members of novel categories more readily than atypical objects (Barrett, 1995). However, there are important differences between the typical and atypical faces and birds tested in this study. The typical and atypical bird exemplars were drawn from different species and therefore differed in their external shape contours and internal features (see Figure 2). In contrast, the typical and atypical human faces differed only with respect to internal properties (i.e. shape and spacing of eye, nose and mouth features). It is remarkable that by 3 years of age, children are sensitive to the subtle perceptual cues that characterize typical and atypical faces.

Unlike their preference for atypical faces and birds, participants failed to show an atypicality bias to cars. This finding stands in direct contrast to the previous study where 50/50 car morphs elicited a strong preference to the atypical vehicle (Tanaka \& Corneille, 2007). These divergent results can perhaps best be explained in terms of the different time periods of the two studies. The typicality ratings and atypicality bias measures reported in the Tanaka and Corneille (2007) paper were obtained in the mid-1990s and it is unlikely that the cars that were judged as typical and atypical by participants during that time are perceived as typical and atypical by contemporary participants. This finding suggests that changing styles in car models alter our conceptions of typicality, which may have perceptual consequences.

The obtained atypicality finding is consistent with Krumhansl's distance-density hypothesis (Krumhansl, 1978) in which the perceived similarity of any two objects is co-determined by their metric distance and the density of surrounding exemplars. As demonstrated in these studies, the 50/50 morph - despite being structurally equidistant from both parent images was perceived to be more similar to its atypical parent than its typical parent. As 
implemented in an attractor network (Tank \& Hopfield, 1987), atypical representations situated in a relatively sparse region of space possess larger attractor fields and capture a broader range of perceptual inputs. In comparison, typical objects located in densely populated areas exhibit compressed fields that will attract a more restricted range of perceptual inputs. In this study, a systematic bias was demonstrated for well-organized perceptual spaces, such as the bias found for atypical faces and birds. However, for perceptual categories that are less coherently organized by the observer, such as cars from the mid-1990s, no systematic preference was observed.

Although the face and bird atypicality bias did not increase with age, it should be possible to induce an atypical preference through category learning. In a recent experiment, we (Kantner \& Tanaka, submitted) created artificial categories composed of typical and atypical polygons. Following a computer algorithm, the typical polygons were constrained to small variations from the category prototype whereas the atypical polygons were permitted to show a larger degree of variation. Prior to category exposure, participants demonstrated no preference for the atypical or typical parent image in the morph task. However, after a brief familiarization session, a reliable atypicality bias emerged where participants judged the $50 / 50$ morph as bearing a stronger resemblance to the atypical over the typical parent. These findings suggest that a relatively small amount of category experience is sufficient to bias the perception of adults for artificial categories. In contrast, by age 3, young children have received enough exposure to faces and birds to show a reliable and stable atypicality bias.

In conclusion, using a novel morphing paradigm we probed the perceptual spaces of faces, birds and cars in children and adults. We found that very young children exhibited a bias for atypical faces and birds that was as robust as the bias shown by older children and adults. These findings suggest that by age 3, children construct perceptual categories for faces and birds that are organized in a similar manner to adults. These categories are not only sensitive to the structural similarities of within-category exemplars, but also to their distribution in the perceptual space.

\section{Acknowledgments}

This study was funded by grants from the James S. McDonnell Foundation, the National Science Foundation (No. SBE-0542013), the National Institutes of Health and the Natural Sciences and Engineering Research Council of Canada to JWT and a University of Victoria Undergraduate Research Scholarship to TM.

\section{References}

Anastasi JS, Rhodes MG. An own-age bias in face recognition for children and older adults. Psychonomic Bulletin \& Review. 2005; 12:1043-1047. [PubMed: 16615326]

Barrett, M. Early lexical development. In: Fletcher, Paul; MacWhinney, Brian, editors. The handbook of child language. Blackwell; Oxford: 1995. p. 362-392.

Bartlett JC, Hurry S, Thorley W. Typicality and familiarity of faces. Memory \& Cognition. 1984; 12:219-228.

Carey S, Diamond R. Are faces perceived as configurations more by adults than by children? Visual Cognition. 1994; 1:253-274.

Chang PP, Levine SC, Benson PJ. Children's recognition of caricatures. Developmental Psychology. 2002; 38:1038-1051. [PubMed: 12428713]

Crookes K, McKone E. Early maturity of face recognition: no childhood development of holistic processing, novel face encoding, or face-space. Cognition. 2009; 111:219-247. [PubMed: 19296930]

Going M, Read JD. Effects of uniqueness, sex of subject, and sex of photograph on facial recognition. Perceptual \& Motor Skills. 1974; 39:109-110. 
Humphreys K, Johnson MH. The development of 'face-space' in infancy. Visual Cogniton. 2007; 15:578-598.

Johnston RA, Ellis HD. Age effects in the processing of typical and distinctive faces. Quarterly Journal of Experimental Psychology A. 1995; 48:447-465.

Kantner J, Tanaka JW. Category learning produces the atypicality bias in object perception. submitted.

Krumhansl CL. Concerning the applicability of geometric models to similarity data: the interrelationship between similiarity and spatial density. Psychological Review. 1978; 85:445-463.

Lewis MB. Face-space-R: towards a unified account of face recognition. Visual Cognition. 2004; 11:29-69.

Light LL, Kayra-Stuart F, Hollander S. Recognition memory for typical and unusual faces. Journal of Experimental Psychology: Human Learning and Memory. 1979; 5:212-228. [PubMed: 528913]

Meytlis M, Sirovich L. On the dimensionality of face space. IEEE Transactions of Pattern Analysis and Machine Intelligence. 2007; 29:1262-1267.

Mondloch CJ, Le Grand R, Maurer D. Configural face processing develops more slowly than featural face processing. Perception. 2002; 31:553-566. [PubMed: 12044096]

Nishimura M, Maurer D, Gao X. Exploring children's face-space: a multidimensional scaling analysis of the mental representation of facial identity. Journal of Experimental Child Psychology. 2009; 103:355-375. [PubMed: 19342062]

O'Toole AJ, Deffenbacher KA, Valentin D, Abdi H. Structural aspects of face recognition and the otherrace effect. Memory \& Cognition. 1994; 22:208-224.

Pellicano E, Rhodes G. Holistic processing of faces in preschool children and adults. Psychological Science. 2003; 14:618-622. [PubMed: 14629695]

Quinn PC. The categorical representation of visual pattern information by young infants. Cognition. 1987; 27:147-179.

Quinn, PC. Beyond prototypes: asymmetries in infant categorization and what they teach us about the mechanisms guiding early knowledge acquisition. In: Kail, RV.; Reese, HW., editors. Advances in child development and behavior. Vol. 29. Academic Press; San Diego, CA: 2002. p. 161-193.

Rhodes G, Byatt G, Tremewan T, Kennedy A. Facial distinctiveness and the power of caricatures. Perception. 1997; 26:207-223. [PubMed: 9274754]

Sangrigoli S, de Schonen S. Effect of visual experience on face processing: a developmental study of inversion and non-native effects. Deveopmental Science. 2004; 7:74-87.

Southgate V, Meints K. Typicality, naming, and category membership in young children. Cognitive Linguistics. 2001; 11:5-16.

Strauss MS. Abstraction of prototypical information by adults and 10-month-old infants. Journal of Experimental Psychology: Human Learning and Memory. 1979; 5:618-632. [PubMed: 528918]

Tanaka JW, Corneille O. Typicality effects in face and object perception: further evidence for the attractor field model. Perception \& Psychophysics. 2007; 69:619-627. [PubMed: 17727115]

Tanaka JW, Kay JB, Grinnell E, Stansfield B, Szechter L. Face recognition in young children: when the whole is greater than the sum of its parts. Visual Cognition. 1998a; 5:479-496.

Tanaka JW, Giles M, Kremen S, Simon V. Mapping attractor fields in face space: the atypicality bias in face recognition. Cognition. 1998b; 68:199-220. [PubMed: 9852665]

Tank DW, Hopfield JJ. Collective computation in neuron like circuits. Scientific American. 1987; 257:104-114. [PubMed: 3452925]

Valentine T. A unified account of the effects of distinctiveness, inversion, and race in face recognition. Quarterly Journal of Experimental Psychology. 1991; 43A:161-204. [PubMed: 1866456]

Valentine T, Bruce V. The effect of race inversion and encoding activity upon face recognition. Acta Psychologica. 1986; 61:259-273. [PubMed: 3716859]

Vokey JR, Read JD. Familiarity, memorability, and the effect of typicality on the recognition of faces. Memory \& Cognition. 1992; 20:291-302.

Willenbockel V, Sadr J, Fiset D, Horne G, Gosselin F, Tanaka JW. Controlling low-level image properties: the SHINE toolbox. Behavioral Research Methods. in press.

Wilson HR, Loffler G, Wilkinson F. Synthetic faces, face cubes, and the geometry of face space. Vision Research. 2002; 42:2909-2923. [PubMed: 12450502] 


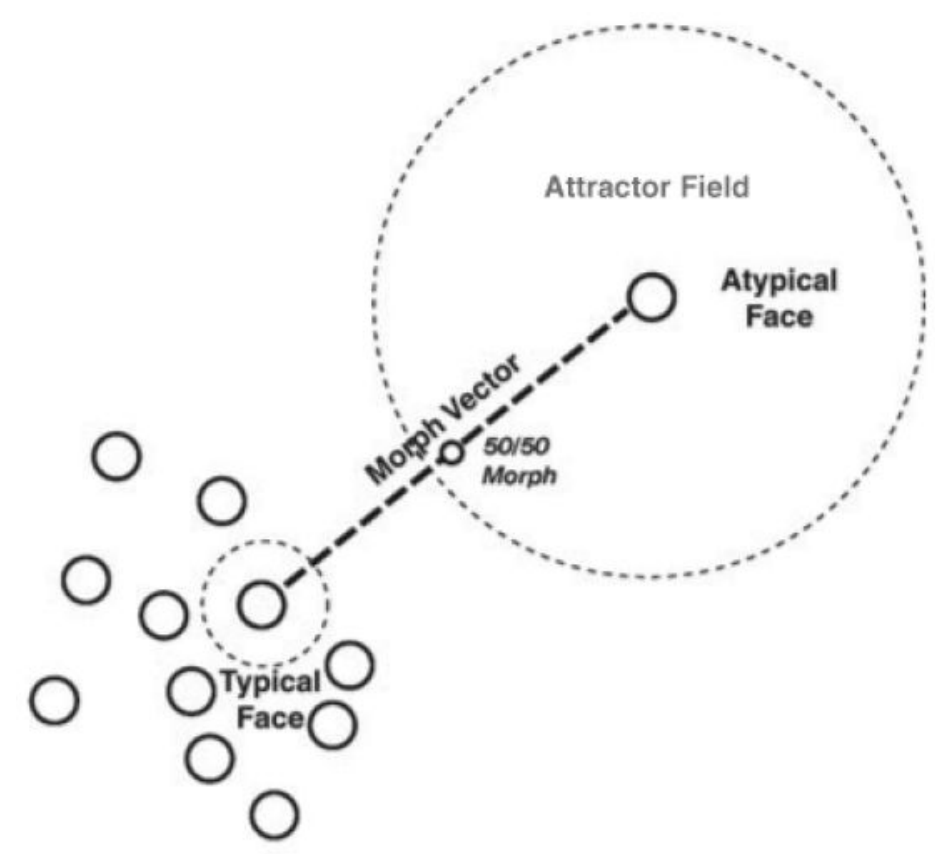

$\mathrm{O}$

Figure 1.

Diagram of the attractor field model. The atypical and typical representations of interest are depicted as filled grey circles. Located in a sparse sub-region of face space, the atypical face representation has a larger attractor field relative to the smaller attractor field of the typical representation situated in a denser sub-region. The morph vector is indicated by the dashed line connecting the atypical and typical parent face representations, and morph representations are located along the vector. The 50/50 morph is located at the midpoint of the vector and is equal in its physical distance from its atypical and typical parent representations. The atypicality bias is the result of the 50/50 morph lying closer to attractor boundary of the atypical representation than the typical representation. 

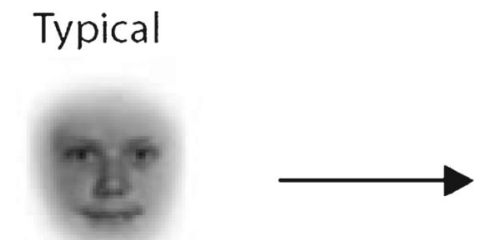

50/50 Morph
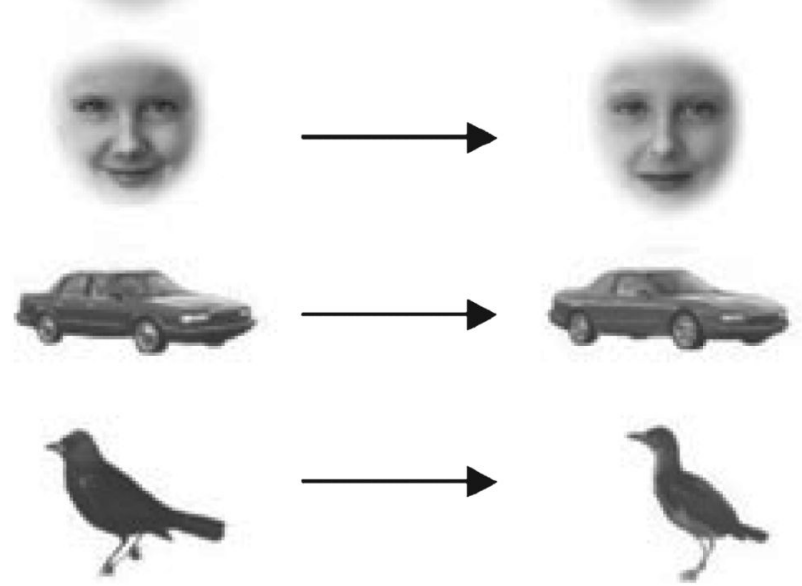
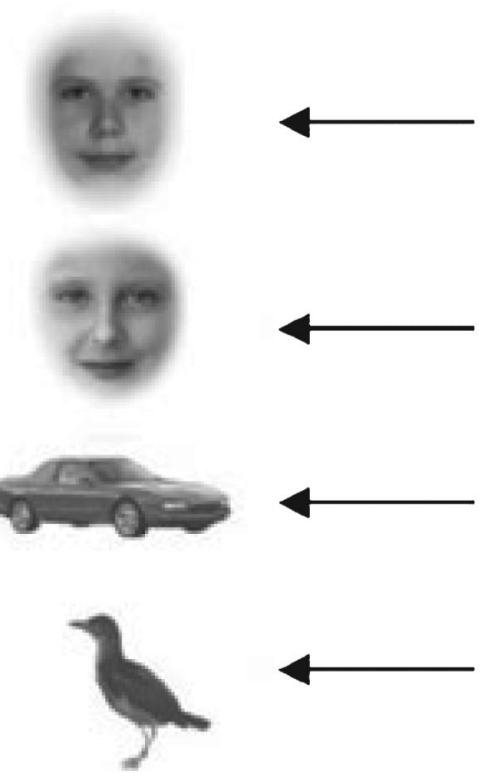

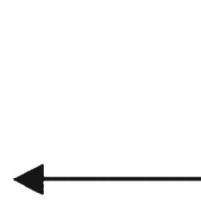

Atypical
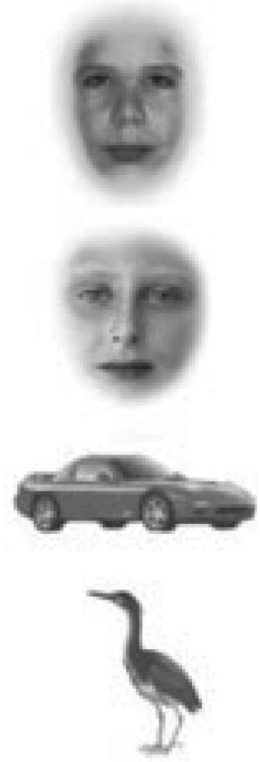

Figure 2.

Examples of 50/50 morphs between typical and atypical children's faces (first two rows), typical and atypical cars (third row) and typical and atypical birds (fourth row). 


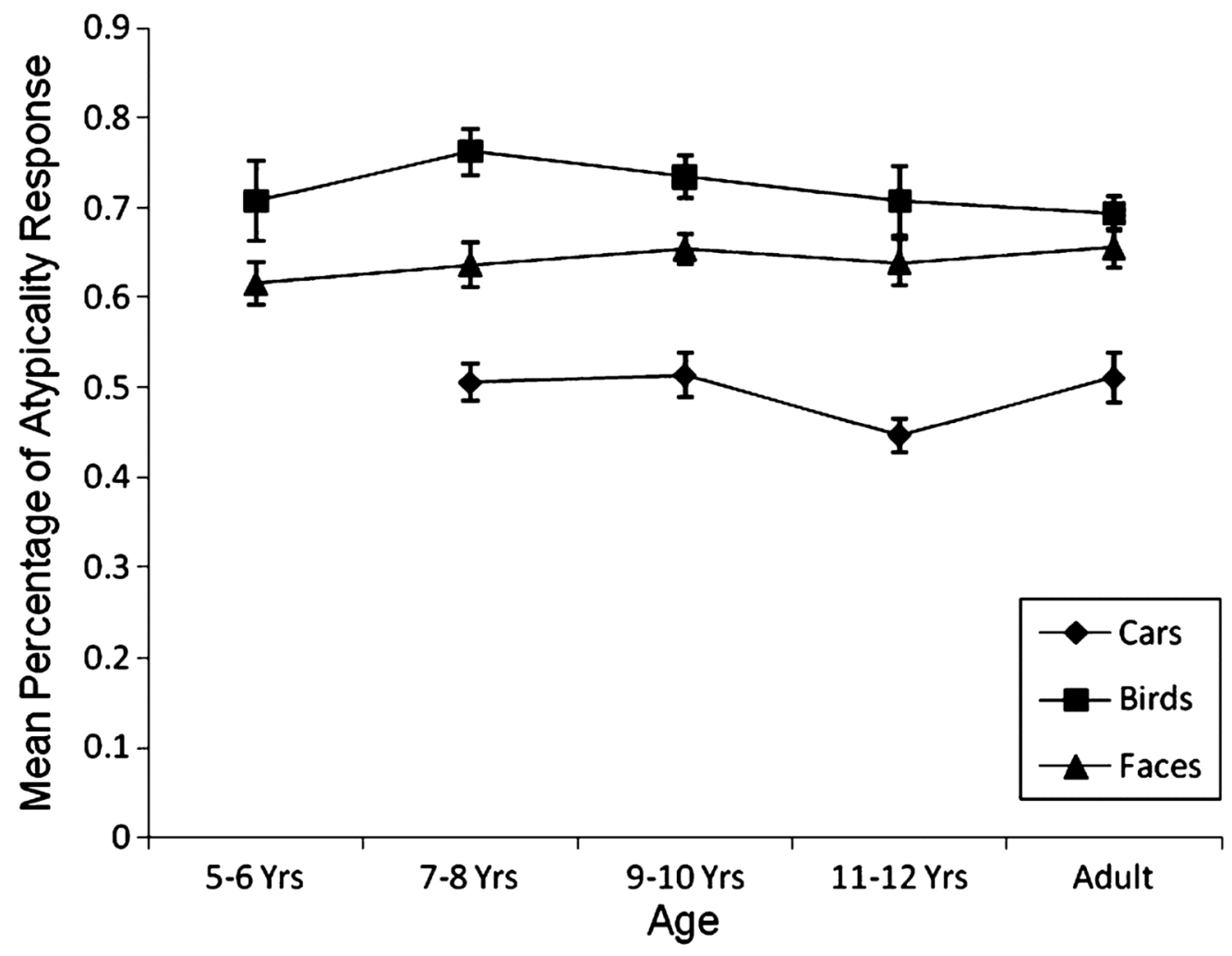

Figure 3.

Percent atypicality responses for 3-4-year-olds, 5-6-year-olds, 7-8-year-olds, 9-10-yearolds, 11-12-year-olds and adults for faces, cars and birds. The dashed line indicates the $50 \%$ unbiased level. 